\title{
Large parathyroid adenoma presenting as a femoral fracture in a young male
}

\author{
Duncan Light ${ }^{*}$, Chakri Munipalle, Vijay Kurup
}

General Surgery, North Tees and Hartlepool NHS Foundation Trust, Stockton on Tees, UK;

*Corresponding Author: duncan.light@hotmail.co.uk

Received 16 September 2013; revised 13 October 2013; accepted 2 November 2013

Copyright (C) 2013 Duncan Light et al. This is an open access article distributed under the Creative Commons Attribution License, which permits unrestricted use, distribution, and reproduction in any medium, provided the original work is properly cited.

\section{ABSTRACT}

Parathyroid adenoma classically presents symptoms of hypercalcaemia. Here, we present a case of a rare presentation of a parathyroid adenoma in a young male patient with a pathological fracture.

Keywords: Parathyroid Adenoma; Hypercalcaemia; Femoral Fracture

\section{INTRODUCTION}

Osteoporosis secondary to hyperparathyroidism is a rare presentation in young patients. The case study below illustrates a case of a young male who presents a pathological fracture secondary to giant parathyroid adenoma.

\section{CASE PRESENTATION}

A 27-years-old male presented to the emergency department with femoral fracture following a fall onto his left hip (Figure 1). He had been previously fit and well though he had developed increasing thirst and lethargy in the preceeding weeks. He was of normal build and nutritional status. He underwent a femoral nail the following day. Initial investigations revealed a corrected calcium of 3.32 and PTH of 2.7. Other biochemical tests were normal. Sestamibi scan showed a likely parathyroid adenoma in the right inferior pole of the thyroid gland (Figure 2). An ultrasound of his neck confirmed the finding. He was taken for neck exploration where the diagnosis was confirmed and a large parathyroid adenoma weighing 4 grams was removed (Figures 3 and 4). Histology did not reveal any evidence of malignancy and subsequent phenotyping excluded the presence of MEN syndrome. Over the subsequent weeks his calcium level rose and at 6 months follow-up his calcium level had returned to normal and he was asymptomatic.

\section{DISCUSSION}

Hyperparathyroidism commonly presents with symptoms of hypercalcaemia such as abdominal pain, bone pain, fatigue, depression and nephrolithiasis. It can be classified into three main groups. Primary hyperparathyroidism is due to parathyroid adenoma in $85 \%$ of patients, the remainder are due to multiple adenomas and parathyroid cancers. It affects 1 in 2000 men annually. Secondary hyperparathyroidism is due to chronic vitamin $\mathrm{D}$ deficiency as a result of chronic renal failure. Tertiary hyperparathyroidism is a result of autonomous parathyroid stimulation following a period of persistent parathyroid stimulation. Biochemical investigations reveal raised calcium, raised PTH and decrease in phosphate levels in the blood. Urinary calcium will be low in comparison to

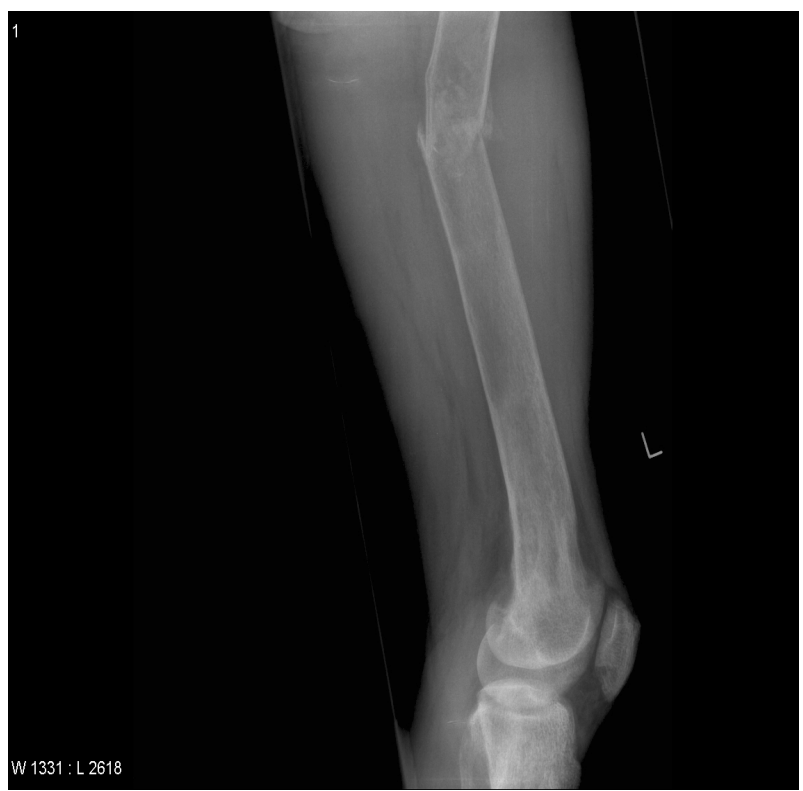

Figure 1. Femoral fracture. 


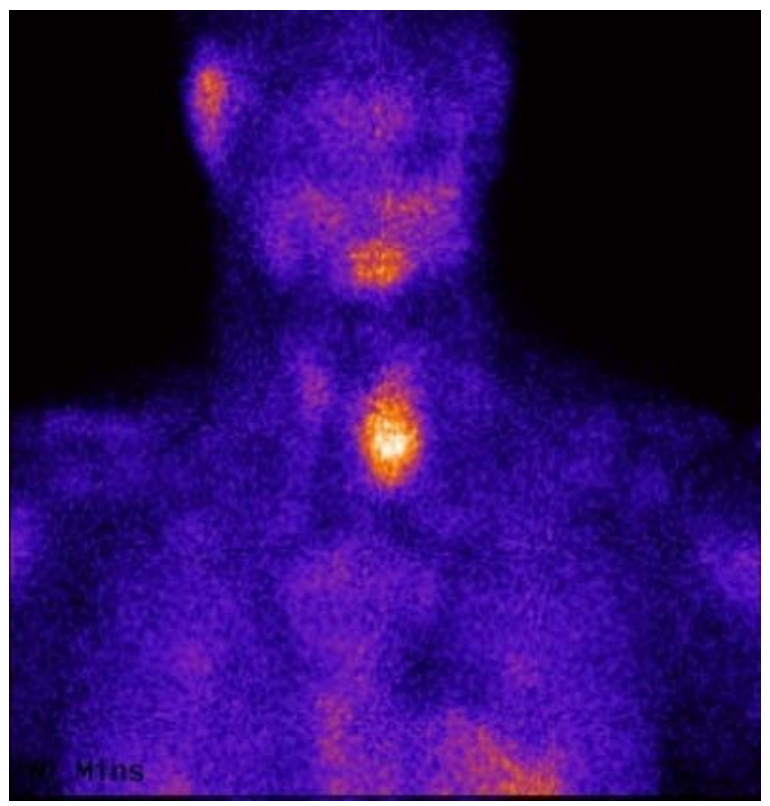

Figure 2. Sestamibi scan.

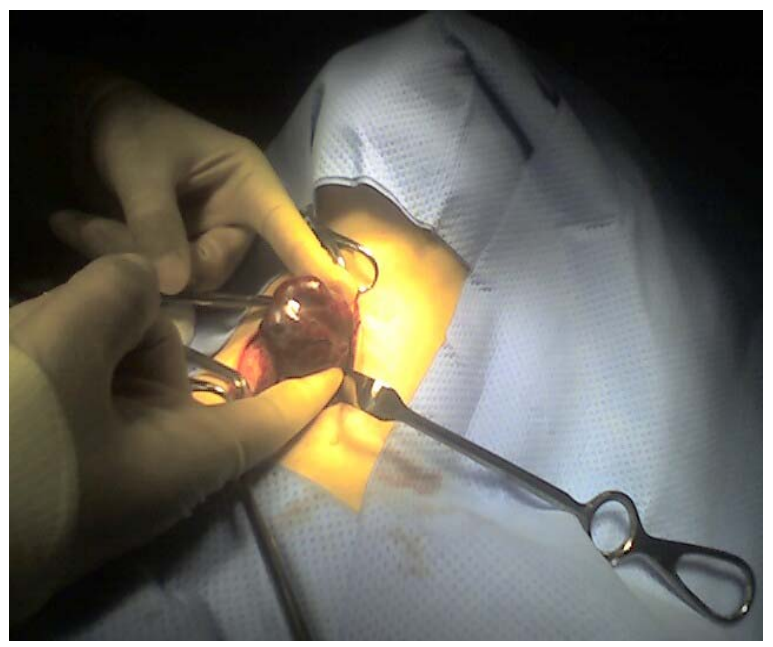

Figure 3. Excision of a large parathyroid adenoma.

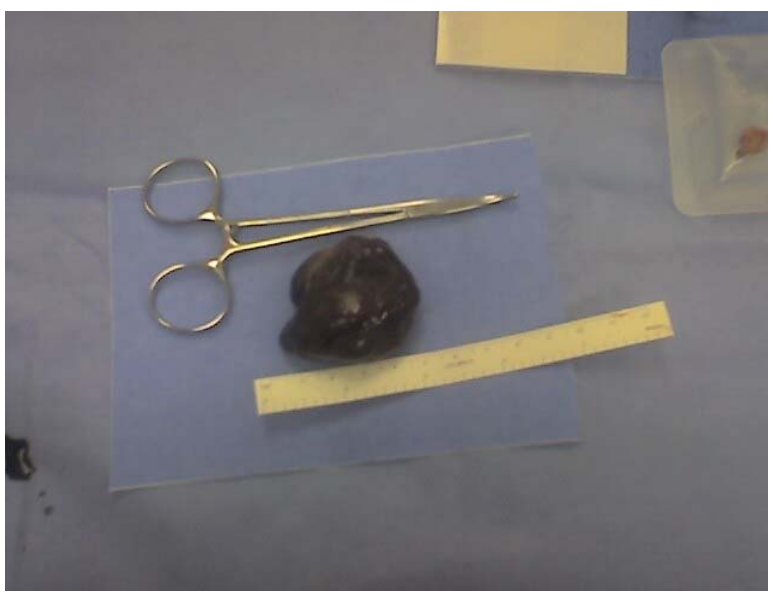

Figure 4. Excised parathyroid adenoma. familial hypercalcaemia hypocalciuria.

Pathological fractures are rare in young adults. A pathological fracture is defined as a fracture which occurs without adequate trauma and is caused by a preexistent pathological bone lesion. Causes include resorption of bone mass (osteoporosis, hyperpara-thyroidism), reduction of bone quality (osteomalacia, osteonecrosis), insufficient bone production (osteogenesis imperfecta, fibrous dysplasia), augmented bone resorption (giant cell granulomas, aneurysmal bone cyst), pathological bone remodelling (Paget's disease) or local bone destruction due to malignancy. It must be detected clinically as well as radiologically and its cause diagnosed histologically in order to ensure adequate therapy.

Pathological fracture in severe hyperparathyroidism can be associated with osteitis fibrosa cystica. It can be seen as a classical skeletal complication of hyperparathyroidism in some patients, although it is rare in the modern era. It occurs in approximately $2 \%$ of individuals diagnosed with hyperparathyroidism [1]. It is associated with a loss of bone mass due to increased osteoclast activity in the matrix secondary to raised PTH. In addition, there is experimental evidence that elevated PTH secretion may be implicated in the enhanced degradation of 25-hydroxy-vitamin D which could contribute to the formation of osteoporosis. As a result there is a weakening of the bones as their calcified supporting structures are replaced with fibrous tissue and the formation of cystlike brown tumors in and around the bone increasing the risk of fracture. A number of studies have confirmed that there is a both an increased risk of fracture in patients with primary hyperparathyroidism and a reduced risk of fractures in patients who undergo parathyroidectomy [2].

The use of preoperative localisation studies is controversial. Main methods of imaging are ultrasound, computed tomography and nuclear imaging. Technetium Tc $99 \mathrm{~m}$ sestamibi is the radio-pharmacological agent of choice, with a sensitivity reported to be between $72 \%$ $100 \%$ [3]. One reason for the controversy around sestamibi is the variable quality and accuracy of scans between different units. This is also countered by the fact that studies have shown that experienced surgeons have a $90 \%$ - 95\% cure rate in patients who undergo neck exploration for the first time. A recent study has shown that sestamibi combined with ultrasound increases the accuracy of detection for parathyroid adenomas [4].

Surgical management is the main modality of treatment in parathyroid adenoma. It involves resection of the parathyroid gland by traditional neck exploration or minimally invasive parathyroidectomy. As yet there is no definitive evidence for the benefit of either technique over the other. There is however a growing trend towards minimally invasive surgery either through preoperatively localised single gland excision or intraoperative locali- 
sation. Indeed, in a number of asian countries there is a growing trend towards parathyroid surgery via remote endoscopic access through the axilla and chest due to patients reluctance to have incisions visible in the neck [5].

Another facet of modern parathyroid surgery is intraoperative monitoring of parathyroid hormone levels. Due to the short half-life of parathyroid hormone in the blood stream, it is possible to assess operative success with serum PTH levels intra-operatively. It is also possible to perform recurrent laryngeal monitoring intraoperatively although this is still controversial and neither technique has become standard practice.

\section{CONCLUSION}

This case illustrates a rare presentation of a large parathyroid adenoma in a young patient and the successful management of complications with surgery.

\section{REFERENCES}

[1] Kronenberg, H.M., Schlomo, M., Polansky, K.S. and Larsen, P.R. (2008) The parathyroid glands, hypercalcemia, and hypocalcemia. Williams textbook of endocrinology. 11th Edition, WB Saunders, St. Louis, Chapter 266.
[2] VanderWalde, L.H., Liu, I.L. and Haigh, P.I. (2009) Effect of bone mineral density and parathyroidectomy on fracture risk in primary hyperparathyroidism. World Journal of Surgery, 33, 406-411.

[3] Shen, W., Sabanci, U., Morita, E.T., Siperstein, A.E., Duh, Q.Y. and Clark, O.H. (1997) Sestamibi scanning is inadequate for directing unilateral neck exploration for first-time parathyroidectomy. Archives of Surgery, 132, 969-976.

http://dx.doi.org/10.1001/archsurg.1997.01430330035005

[4] Patel, C.N., Salahudeen, H.M., Lansdown, M. and Scarsbrook, A.F. (2010) Clinical utility of ultrasound and 99 mTc sestamibi SPECT/CT for preoperative localization of parathyroid adenoma in patients with primary hyperparathyroidism. Clinical Radiology, 65, 278-287.

[5] Lang, B. (2009) Minimally invasive thyroid and parathyroid surgery.

http://www.fmshk.org/database/articles/03mb2_6.pdf 